\title{
Pathogenetic Subtypes and Ischemic Stroke Outcomes in Patients with Chronic Kidney Disease
}

\author{
Sargylana A. Chugunova ${ }^{1, *}$ and Tuyara S. Egorova ${ }^{2}$
}

\author{
${ }^{1}$ M.K. Ammosov North-Eastern Federal University, 27, Oyunsky St, Yakutsk, 677000, Russia \\ ${ }^{2}$ Republic Hospital No. 2 - Emergency Center, 83A, P. Alekseeva St., Yakutsk, 677005, Russia \\ Corresponding author. Email: sa.chugunova@mail.ru
}

\begin{abstract}
A study has been conducted to investigate the relationships between the risk of developing various pathogenetic subtypes of ischemic stroke (IS) and its outcomes with chronic kidney disease (CKD). A retrospective study was conducted in the group of IS patients $(n=1070)$ : with CKD $(n=343)$ and without CKD $(n=727)$. TOAST classification was used to determine IS subtypes. The study data showed that in patients with CKD compared to patients without CKD, TOAST2 frequency was more common in total group $(\mathrm{p}<0.0001 ; \mathrm{OR}=1.878$ (1.423-2.478)), among men $(\mathrm{p}<0.001 ; \mathrm{OR}=2.008(1.370-2.943))$ and among women $(\mathrm{p}=0.009$; $\mathrm{OR}=1.720(1.145-2.584))$; TOAST1 frequency was rare in total group $(\mathrm{p}<0.0001 ; \mathrm{OR}=0.565(0.435-0.734))$, among men $(\mathrm{p}<0.0001 ; \mathrm{OR}=0.502(0.353-0.713))$ and among women $(\mathrm{p}=0.042 ; \mathrm{OR}=0.665(0.448-0.487))$; stroke manifestations were more severe an NIHSS scale ( $\mathrm{p}=0.0001)$, Glasgow coma scale ( $\mathrm{p}=0.002)$; IS mortality was higher $(13.7 \%$ versus $7.4 \%$; $=0.001$; $\mathrm{OR}=1.979(1.308-2.994)$ in total group and TOAST2 mortality was higher $(25.0 \%$ vs.14.9 \%; p=0.027; OR=1.910 (1.072-3.404) in total group and among women (29.7\% versus $11.1 \%$; $=0.004 ; \mathrm{OR}=3.378$ (1.446-7.890)). Conclusions. IS patients with CKD are more likely to develop cardioembolic stroke $(\mathrm{p}<0.0001$; $\mathrm{OR}=1.878(1.423-2.478)$ ), have more severe stroke manifestations (NIHSS score, $\mathrm{p}=0.0001$; Glasgow score, $\mathrm{p}=0.002)$, have higher cardioembolic stroke mortality among women $(29.7 \%$ versus $11.1 \% ; \mathrm{p}=0.004 ; \mathrm{OR}=3.378(1.446-7.890)$ compared to IS patients without $\mathrm{CKD}$.
\end{abstract}

Keywords: pathogenetic subtypes, ischemic stroke outcomes, chronic kidney disease

\section{INTRODUCTION}

Chronic kidney disease (CKD) is a common pathology that affects up to a third of the population aged over 75 [1].

CKD is diagnosed in the presence of kidney injury and/or decreased glomerular filtration that persists for three or more months [2, 3]. Kidney damage is diagnosed in case of any manifestation of pathological albuminuria/protheinuria, pathological urine precipitate, histological signs of kidney damage, presence of pathology based on X-ray diagnostics results [3]. In CKD the glomerular filtration rate decrease is less than $60 \mathrm{ml} / \mathrm{min} / 1.73 \mathrm{~m}^{2}[2,3]$ and it is the main marker of this pathological state. The CKD stage is estimated by the calculated glomerular filtration rate [3].
Chronic kidney disease is recognized as a risk factor for cardiovascular disease (CVD) along with other factors as arterial hypertension, diabetes mellitus and dyslipidemia [4, 5]. Patients with reduced glomerular filtration rate (GFR) less than $59 \mathrm{ml} / \mathrm{min} / 1.73 \mathrm{~m}^{2}$ have a high risk of cardiovascular disease, and patients with GFR less than $30 \mathrm{ml} / \mathrm{min} / 1.73 \mathrm{~m}^{2}$ have a very high risk of CVD [6]. Chronic kidney disease is an independent predictor of ischemic heart disease outcomes [7].

Recently many studies have been devoted to the study of CKD association with cerebrovascular diseases $[1,8,9]$. Chronic kidney disease, defined as reduced glomerular filtration rate or increased urinary albumin excretion, has been proven to be a risk factor for cerebrovascular diseases [8]. CKD is a risk factor for the development of subclinical cerebrovascular pathological states and cognitive impairment, the brain white matter pathology, microbleeds $[1,5,8,10]$. The 
mechanisms responsible for these associations are currently insufficiently studied [1].

Moreover, chronic kidney disease is associated with an increased risk of stroke [4, 8]. Disorders of kidney function and CKD are common in patients with ischemic stroke (IS) and transient ischemic attacks (TIA) $[5,11,12]$. According to Kelly D. M. et al. study (2020) [12], CKD were diagnosed in $40 \%$ of 3178 patients, including transient ischemic attack $(n=1167)$, ischemic stroke $(\mathrm{n}=1802)$ and intracerebral hemorrhage $(n=209)$. The kidney dysfunction was observed in $44.6 \%$ of patients with IS and TIA [11]. Patients with a calculated glomerular filtration rate $<60 \mathrm{ml} / \mathrm{min} / 1.73 \mathrm{~m}^{2}$ or proteinuria make up $46 \%$ of the total ischemic stroke patients and $39 \%$ of the total intracerebral hemorrhage patients [5].

Reduced glomerular filtration rates have been found to increase stroke risk by more than $40 \%$, and proteinuria increases this risk by more than $70 \%[1,5]$. Beyond the original meaning of CKD as high potential risk for hemodialysis need, the chronic kidney disease is risk factor for CVD [5]. Patients with ischemic stroke should be considered as a high-risk group for CKD and need active prevention of this pathological condition [13].

Thus, CKD has a strong two-way relationships with stroke risk, but the mechanisms underlying this association have not been fully explained. A few studies have been conducted to investigate various etiological stroke subtypes in CKD [12]. Research of ischemic stroke pathogenetic subtypes will contribute to understanding the mechanisms of acute cerebral circulation disorders in chronic kidney disease.

The purpose of the study is to examine the relationships between the risk of ischemic stroke's different pathogenetic subtypes and its outcomes with chronic kidney disease.

\section{METHODS AND MATERIALS}

A retrospective study was conducted in a group of 1,070 patients with ischemic stroke (IS) hospitalized at the Regional Vascular Center of "Republic Hospital No. 2 - Emergency Center" (Yakutsk).

The study group consisted of 2 subgroups: the 1st subgroup ( $\mathrm{n}=343)$ included ischemic stroke (IS) patients with diagnosed chronic kidney disease (CKD) which were hospitalized in 2017-2019; the 2nd subgroup $(n=727)$ included IS patients without CKD, consecutively hospitalized in 2019.

In all cases included in the study, stroke diagnosis and IS subtype were established by neurological examination; case history; neuroimaging (brain CT or brain MRI); ultrasound of cerebral arteries and heart, electrocardiography, Holter monitoring, laboratory examinations. The IS pathogenetic subtype was determined in accordance with the TOAST criteria [14]: TOAST 1 - stroke of large arteries atherosclerosis; TOAST 2 - cardioembolic etiology stroke; TOAST 3 occlusion of small cerebral vessels; TOAST 4 - stroke of other determined etiology; TOAST 5 is a stroke of undetermined etiology.

The CKD diagnosis was established by criteria based on the glomerular filtration rate and albuminuria measurement, therapist examination, case history, laboratory data, ultrasound studies of parenchymal organs. The CKD degree was diagnosed according to the criteria of KDIGO clinical recommendations (2012) [2]. Between the study subgroups a comparative analysis of the following parameters was performed: age, sex, stroke subtype, neurological manifestations an NIHSS scale and Glasgow coma scale, stroke outcomes (fatal and non-fatal outcomes).

Statistical analyses were performed by Statistica 13.0. Quantitative variables were described by mean and standard deviation $(\mathrm{M} \pm \mathrm{SD})$. The comparison of the quantitative variables in two independent groups was carried out by t-test. Frequency comparisons in two independent groups were performed with Pearson's test, $\chi^{2}$, Fisher's exact test. The odds ratio (OR) and $95 \%$ confidence interval (CI) were calculated for significant differences. The null hypothesis was rejected at $\mathrm{p}<0.05$.

\section{RESEARCH RESULTS}

In the total patient group $(\mathrm{n}=1070)$, male patients were $58.1 \%(n=622)$, female patients $-41.9 \%(n=448)$. The mean age of IS patients was $65.5 \pm 12.7$ years. The mean age of male patients was statistically significant lower than one of female patients $(64.5 \pm 11.9$ versus $69.9 \pm 12.3$ years $)(\mathrm{p}=0.0001)$

The neurological symptom's severity in the disease debut did not have significant differences between male and female patients: the NIHSS score was $8.3 \pm 8.3$ points in the male group, $9.3 \pm 9.2$ points in the female group ( $\mathrm{p}=0.060)$; Glasgow score was $13.9 \pm 4.1$ and $13.7 \pm 4.7$ points, respectively $(\mathrm{p}=0.430)$.

According to TOAST classification, the ischemic stroke types in the total group were presented as follows: TOAST 1 (atherotrombotic type) was diagnosed in 661 patients, including 407 men $(65.4 \%$ of total IS cases among men) and 254 women $(56.7 \%$ of total IS cases among women); TOAST 2 (cardioembolic type) - 303 IS cases, including 149 men (23.9\%) and 154 women (34.4\%); TOAST 3 (lacunar type) 66 cases, including 39 men $(6.3 \%)$ and 27 women $(6.0 \%)$ : TOAST $4-29$ cases, including 20 men $(3.2 \%)$ and 9 women $(2.01 \%)$; TOAST 5 - 11 cases, including 7 men $(1.1 \%)$ and 4 women $(0.9 \%)$. 
In the 1st subgroup, type TOAST 1 accounted for the majority of cases -180 (52.5\% of all IS cases in this subgroup), including -105 men (54.1\% of male patients of the 1 st subgroup), 75 women (50.3\% of female patients of the 1 st subgroup) $(p=0.656)$. In the 1st subgroup the cardioembolic stroke (TOAST 2) accounted for more than a third of all cases - 128 cases (37.3\% of the total IS cases in this subgroup), including 64 men $(32.9 \%), 64$ women $(42.9 \%)(\mathrm{p}=0.071)$. Lacunar strokes (subtype TOAST 3) accounted for 27 cases ( $7.9 \%$ of the total IS cases in this subgroup), including 18 men $(9.3 \%), 9$ women $(6.0 \%)$; $(\mathrm{p}=0.256)$. Ischemic strokes of other determined etiology (subtype TOAST 4) accounted for 7 cases $(2.0 \%)$, including 6 men $(3.1 \%) ; 1$ woman $(0.7 \%)$ $(\mathrm{p}=0.142)$. Ischemic strokes of undetermined etiology (TOAST 5 subtype) accounted for 1 case (men $0(0 \%)$, women $1(0.7 \%) ; \mathrm{p}=0.437)$. Thus, no significant differences have been established in the IS pathogenetic subtypes distribution between men and women with chronic kidney disease.

In the $2 \mathrm{~d}$ subgroup, type TOAST1 was diagnosed in 481 patients (66.1\% of all IS cases of this subgroup), including 302 men $(70.4 \%$ of male patients of the $2 \mathrm{~d}$ subgroup), 179 in women (60.1\% of female patients of the $2 \mathrm{~d}$ subgroup) $\left(\mathrm{p}=0.004 ; \chi^{2}=8.380, \mathrm{df}=1\right.$; $\mathrm{OR}=1.581 ; 95 \%$ CI: $1.158-2.158)$.

Strokes of cardioembolic etiology (TOAST2) accounted for 175 cases $(24.1 \%$ of the total IS in the $2 \mathrm{~d}$ subgroup), including 85 men (19.8\%), 90 women $(30.2 \%)(\mathrm{p}=0.001 ; \chi 2=10.382, \mathrm{df}=1 ; \mathrm{OR}=0.571$; $95 \%$ CI: 0.405-0.805). Lacunar strokes (subtype TOAST 3) accounted for 39 cases (5.4\% of the total IS of the $2 \mathrm{~d}$ subgroup), including 21 men (4.9\%), 18 women $(6.0 \%) ;\left(\mathrm{p}=0.500 ; \chi^{2}=0.454, \mathrm{df}=1\right)$. Ischemic strokes of other determined etiology (subtype TOAST 4) amounted to 22 cases (3.02\%), including 14 men $(3.3 \%) ; 8$ women $(2.7 \%)(\mathrm{p}=0.654 ; \chi 2=0.201$, $\mathrm{df}=1$ ). Ischemic strokes of undetermined etiology (TOAST 5 subtype) diagnosed in 10 cases $(1.4 \%)$ $\left(7\right.$ men $(1.6 \%), 3$ women $(1.0 \%) ; \mathrm{p}=0.477 ; \chi^{2}=$ $0.506, \mathrm{df}=1)$.

The CKD stages diagnosed in patients with chronic kidney diseases are: Stage $1-9$ cases $(2.6 \%)$, including 7 men $(3.6 \%), 2$ women $(1.3 \%)(\mathrm{p}=0.308)$; Stage $2 \mathrm{CKD}-78$ cases $(22.7 \%)$, including 55 men $(28.5 \%)$, 23 women $(15.3 \%)(\mathrm{p}=0.004 ; \chi 2=8.325 ; \mathrm{df}=1)$; Stage 3 CKD - 189 cases (55.1\%), including 99 men $(51.3 \%), 90$ women $(60.0 \%)(\mathrm{p}=0.108 ; \chi 2=2.585$; $\mathrm{df}=1)$; Stage $4 \mathrm{CKD}-32$ cases $(9.3 \%)$, among male patients -14 cases $(7.3 \%)$, among female patients 18 cases $(12.0 \%)\left(\mathrm{p}=0.134 ; \chi^{2}=2.248 ; \mathrm{df}=1\right)$; Stage 5 CKD -35 cases $(10.2 \%)$, including 18 men $(9.3 \%)$, 17 women $(11.3 \%)\left(\mathrm{p}=0.408 ; \chi^{2}=0.684 ; \mathrm{df}=1\right)$.
Table 1. Ischemic stroke subtypes in patients with/without chronic kidney disease

\begin{tabular}{|c|c|c|c|}
\hline IS type n (\%) & $\begin{array}{l}\text { 1st subgroup, } \\
\text { IS patients } \\
\text { with CKD, n } \\
(\%)\end{array}$ & \begin{tabular}{|l|} 
2d subgroup, \\
Is patients \\
without CKD, \\
$n(\%)$
\end{tabular} & $\bar{p}$ \\
\hline \multicolumn{4}{|c|}{ total group $(n=1070)$} \\
\hline all types & $343(100)$ & 727 (100) & \\
\hline TOAST1 & $180(52.5)$ & $481(66.1)$ & $<0.0001$ \\
\hline TOAST2 & $128(37.3)$ & 175 (24.1) & $<0.0001$ \\
\hline TOAST3 & $27(7.9)$ & $39(5.4)$ & 0.112 \\
\hline TOAST4 & $7(2.04)$ & $22(3.02)$ & 0.354 \\
\hline TOAST5 & $1(0.3)$ & $10(1.4)$ & 0.190 \\
\hline age, years & $68.7 \pm 12.4$ & $64.1 \pm 12.5$ & $<0.0001$ \\
\hline NIHSS, points & $10.2 \pm 8.2$ & $8,03 \pm 8.8$ & 0.0001 \\
\hline Glasgow, points & $13.3 \pm 2.5$ & $14,1 \pm 4.9$ & 0.002 \\
\hline \multicolumn{4}{|c|}{ male group $(n=622)$} \\
\hline all types & $193(100)$ & $429(100)$ & \\
\hline TOAST1 & $105(54.4)$ & $302(70.4)$ & $<0.0001$ \\
\hline TOAST2 & $64(33.1)$ & $85(19.8)$ & $<0.0001$ \\
\hline TOAST3 & $18(9.3)$ & $21(4.9)$ & 0.035 \\
\hline TOAST4 & $6(3.1)$ & $14(3.3)$ & 0.919 \\
\hline TOAST5 & $0(0)$ & $7(1.6)$ & 0.106 \\
\hline age, years & $65.7 \pm 12.3$ & $61.03 \pm 11.6$ & $<0.0001$ \\
\hline NIHSS, points & $9.5 \pm 7.8$ & $7.8 \pm 8.5$ & 0.019 \\
\hline Glasgow, points & $13.4 \pm 2.4$ & $14,2 \pm 4.6$ & 0.022 \\
\hline \multicolumn{4}{|c|}{ female group $(n=448)$} \\
\hline all types & $150(100)$ & $298(100)$ & \\
\hline TOAST1 & $75(50.0)$ & $179(60.1)$ & 0.042 \\
\hline TOAST2 & $64(42.7)$ & $90(30.2)$ & 0.009 \\
\hline TOAST3 & $9(6.0)$ & $18(6.0)$ & 0.987 \\
\hline TOAST4 & $1(0.7)$ & $8(2.7)$ & 0.283 \\
\hline TOAST5 & $1(0.7)$ & $3(1.0)$ & 1.000 \\
\hline age, years & $72.6 \pm 11.6$ & $68.6 \pm 12.5$ & 0.001 \\
\hline NIHSS, points & $11.2 \pm 8.6$ & $8.4 \pm 9.3$ & 0.0003 \\
\hline Glasgow, points & $13.1 \pm 2.6$ & $14,1 \pm 5.5$ & 0.03 \\
\hline
\end{tabular}

The most common stroke risk factor among CKD patients was arterial hypertension $(\mathrm{AH})-333$ patients (97.1\%), including 185 men (95.9\%), 148 women $(98.7 \%)(\mathrm{p}=0.196)$. Diabetes mellitus was diagnosed in 129 cases $(37.6 \%)$, this factor was diagnosed statistically significant more often among women $(\mathrm{n}=74(49.3 \%))$ compared to men $(\mathrm{n}=55(28.5 \%))$ $\left(\mathrm{p}<0.001 ; \chi^{2}=15.616, \mathrm{df}=1\right)$. Coronary heart disease (CHD) was diagnosed in 162 cases (47.2\%), including 84 men $(43.5 \%), 78$ women $(52.0 \%)(p=0.119$; 
$\chi 2=2.433, \mathrm{df}=1)$. Atrial fibrillation (AF) was diagnosed in 128 cases $(37.3 \%)$, including 64 men (32.9\%), 64 women $(42.9 \%)(\mathrm{p}=0.071 ; \chi 2=3.261, \mathrm{df}=1)$.

Ischemic stroke pathogenetic subtypes in the groups with or without chronic kidney disease (CKD) are presented in Table 1. The IS subtypes distribution between subgroups of patients with and without CKD had statistically significant differences: the incidence of atherothrombotic stroke (TOAST1) was lower in the 1st subgroup compared to the $2 \mathrm{~d}$ group $(\mathrm{p}<0.0001 ; \chi 2=18.481$, $\mathrm{df}=1 ; \mathrm{OR}=0.565$; 95 \% CI: 0.435-0.734), while the cardioembolic stroke (TOAST2) incidence was statistically significant higher among patients with CKD ( $<<0.0001 ; \chi 2=20.145$, $\mathrm{df}=1 ; \mathrm{OR}=1.878 ; 95 \% \mathrm{CI}: 1.423-2.478)$ (Table 1). After gender-adjustment, statistically significant differences in IS subtypes frequencies were established both in male and female patient groups.

Among male patients, the atherothrombotic stroke incidence was lower in patients with CKD $(\mathrm{p}<0.0001$; $\left.\chi^{2}=15.052, \mathrm{df}=1 ; \mathrm{OR}=0.502 ; 95 \% \mathrm{CI}: 0.353-0.713\right)$, and the cardioembolic stroke incidence, in contrast, was higher compared to patients without CKD ( $\mathrm{p}=0.001$; $\chi 2=13.018, \mathrm{df}=1 ; \mathrm{OR}=2.008 ; 95 \%$ CI: $1.370-2.943)$.

Among female patients, in CKD group the cardioembolic stroke incidence was higher $(\mathrm{p}=0.009$; $\chi 2=6.873, \mathrm{df}=1 ; \mathrm{OR}=1.720 ; 95 \% \mathrm{CI}: 1.145-2.584)$, and the atherothrombotic stroke incidence was statistically significant lower $(\mathrm{p}=0.042 ; \chi 2=4.119$, $\mathrm{df}=1 ;$ OR $=0.665 ; 95 \% \mathrm{CI}: 0.448-0.487)$ than one among female patients without kidney disfunction (Table 1). It had been not established significant differences for another stroke subtype frequencies between CKD patients and non- CKD patients both in total group, male and female groups.

The mean age of patients with CKD was $68.7 \pm 12.4$ years and it was statistically higher than this one of patients without kidney dysfunction $(64.1 \pm 12.5$ years $)$ ( $\mathrm{p}<0.0001)$. Among male group, the mean age of CKD patients was $65.7 \pm 12.3$ points, the mean age of nonCKD patients was $61.03 \pm 11.6(\mathrm{p}<0.0001)$. In female group, the mean age of CKD patients was $72.6 \pm 11.6$ and it was statistically higher than this one of patients without CKD $(68.6 \pm 12.5)(\mathrm{p}=0.001)($ Table 1$)$.

The stroke manifestations in the disease debut were more severe in CKD patients. In CKD patients, the neurological symptomatology measurement an NIHSS scale was $10.2 \pm 8.2$ points compared to $8.03 \pm 8.8$ points in non-CKD patients $(\mathrm{p}=0.0001)$. Consciousness depression by Glasgow coma scale was statistically significant more severe in the CKD group $(13.3 \pm 2.5$ points $)$ compared to the non-CKD group (14.1 \pm 4.9 points) $(\mathrm{p}=0.002)$.
Table 2. Ischemic stroke outcomes in groups of patients with/without chronic kidney disease

\begin{tabular}{|c|c|c|c|}
\hline $\begin{array}{l}\text { Stroke type } \\
\left(n_{1}^{*} ; n_{2}^{* \star}\right)\end{array}$ & $\begin{array}{l}\text { IS patients with } \\
\text { CKD,fatal } \\
\text { outcomes, n (\%) }\end{array}$ & $\begin{array}{l}\text { IS patients without } \\
\text { CKD, fatal } \\
\text { outcomes, n ( \%) }\end{array}$ & $p$ \\
\hline \multicolumn{4}{|c|}{ total group $(n=1070)$} \\
\hline $\begin{array}{l}\text { all types } \\
(343 ; 727)\end{array}$ & 47 (13.7) & $54(7.4)$ & 0.001 \\
\hline $\begin{array}{l}\text { TOAST1 } \\
(180 ; 481)\end{array}$ & $15(8.3)$ & $28(5.8)$ & 0.244 \\
\hline $\begin{array}{l}\text { TOAST2 } \\
(128 ; 175)\end{array}$ & $32(25.0)$ & $26(14.9)$ & 0.027 \\
\hline $\begin{array}{l}\text { TOAST3 } \\
(27 ; 39)\end{array}$ & $0(0)$ & $0(0)$ & \\
\hline $\begin{array}{l}\text { TOAST4 } \\
(7 ; 22)\end{array}$ & $0(0)$ & $0(0)$ & \\
\hline $\begin{array}{l}\text { TOAST5 } \\
(1 ; 10)\end{array}$ & $0(0)$ & $0(0)$ & \\
\hline \multicolumn{4}{|c|}{ male group $(n=622)$} \\
\hline $\begin{array}{l}\text { all types } \\
(193 ; 429)\end{array}$ & $20(10.4)$ & $35(8.2)$ & 0.370 \\
\hline $\begin{array}{l}\text { TOAST1 } \\
(105 ; 302) \\
\end{array}$ & $7(6,7)$ & $19(6,3)$ & 0.892 \\
\hline $\begin{array}{l}\text { TOAST } 2 \\
(64 ; 85) \\
\end{array}$ & $13(20.3)$ & $16(18.8)$ & 0.820 \\
\hline $\begin{array}{l}\text { TOAST3 } \\
(18 ; 21) \\
\end{array}$ & $0(0)$ & $0(0)$ & \\
\hline $\begin{array}{l}\text { TOAST4 } \\
(6 ; 14) \\
\end{array}$ & $0(0)$ & $0(0)$ & \\
\hline $\begin{array}{l}\text { TOAST5 } \\
(0 ; 7)\end{array}$ & $0(0)$ & $0(0)$ & \\
\hline \multicolumn{4}{|c|}{ female group ( $n=448)$} \\
\hline $\begin{array}{l}\text { all types } \\
(150 ; 298) \\
\end{array}$ & $27(18.0)$ & $19(6.4)$ & $<0.0001$ \\
\hline $\begin{array}{l}\text { TOAST } 1 \\
(75 ; 179)\end{array}$ & $8(10.7)$ & $9(5.02)$ & 0.101 \\
\hline $\begin{array}{l}\text { TOAST2 } \\
(64 ; 90) \\
\end{array}$ & 19 (29.7) & $10(11.1)$ & 0.004 \\
\hline $\begin{array}{l}\text { TOAST3 } \\
(9 ; 18) \\
\end{array}$ & $0(0)$ & $0(0)$ & \\
\hline $\begin{array}{l}\text { TOAST4 } \\
(1 ; 8) \\
\end{array}$ & $0(0)$ & $0(0)$ & \\
\hline $\begin{array}{l}\text { TOAST5 } \\
(1 ; 3)\end{array}$ & $0(0)$ & $0(0)$ & \\
\hline
\end{tabular}


The average NIHSS scale score in CKD male patients (9.5 \pm 7.8 points) was significantly higher than this one in male patients without kidney function ( $7.8 \pm 8.5$ points) $(\mathrm{p}=0.019)$, as well as the consciousness depression severity by Glasgow scale $(13.4 \pm 2.4$ points versus $14.2 \pm 4.6$ points, respectively, $p=0.022)$. Also, in the female group, statistically significant differences were established in NIHSS score (patients with CKD $11.2 \pm 8.6$; patients without CKD $-8.4 \pm 9.3$ points, $\mathrm{p}=0.0003)$ and in Glasgow scale score $(13.1 \pm 2.6$ versus $14.1 \pm 5.5$ points, respectively, $\mathrm{p}=0.03)($ Table 1$)$.

Stroke outcomes are presented in Table 2. The ischemic stroke mortality in acute period among CKD patients was higher and amounted to $13.7 \%$, while this one was $7.4 \%$ among patients without CKD ( $\mathrm{p}=0.001$; $\chi 2=10.734, \mathrm{df}=1 ; \mathrm{OR}=1.979 ; 95 \% \mathrm{CI}: 1.308-2.994)$.

The cardioembolic stroke (TOAST 2) fatal incidence was higher in the CKD group $(25.0 \%)$ compared to the non-CKD group $(14.9 \%)(\mathrm{p}=0.027 ; \chi 2=4.914, \mathrm{df}=1$; $\mathrm{OR}=1.910 ; 95 \% \mathrm{CI}: 1.072-3.404)$. In the other stroke subtypes, no significant differences in mortality rates between the study subgroups were established.

There were no significant differences in the mortality incidence in all IS types between 1 and 2 subgroups among male patients. Among female patients, statistically significant differences in mortality rates were obtained for cardioembolic stroke: $29.7 \%$ in the CKD group versus $11.1 \%$ in the group without kidney dysfunction $(\mathrm{p}=0.004 ; \chi 2=8.444, \mathrm{df}=1$; $\mathrm{OR}=3.378 ; 95 \%$ CI: $1.446-7.890)$, as well as mortality rates for all IS types: $18.0 \%$ versus $6.4 \%$, respectively $(\mathrm{p}<0.001 ; \chi 2=14.633, \mathrm{df}=1 ; \mathrm{OR}=3.223$; $95 \%$ CI: 1.727-6.016) (Table 2).

\section{DISCUSSION}

In this study, statistically significant differences in the TOAST-classified stroke subtypes distribution were established between IS patients with CRD and IS patients without CKD.

The cardioembolic stroke rate (TOAST2) was statistically significantly higher among CKD patients in the total group $(\mathrm{p}<0.0001 ; \mathrm{OR}=1.878(1.423-2.478)$ ), both among men $(\mathrm{p}<0.001 ; \mathrm{OR}=2.008(1.370-2.943))$ and among women $(\mathrm{p}=0.009 ; \mathrm{OR}=1.720(1.145-2.584))$. The cardioembolic stroke incidence was $37.3 \%$ in IS patients with CKD, while this one was $24.1 \%$ in IS patients without CKD. These results are comparable with of Chinda J. et al. study data [15], which revealed a significantly higher incidence of cardiogenic cerebral embolism in the CKD group compared to the non-CKD group (36\% vs. $26 \%, \mathrm{p}<0.05)$. It was also shown that the prevalence of atrial fibrillation (44\% versus $21 \%$, $\mathrm{p}<0.01)$ and the case history of cardiovascular disease (37\% versus $19 \%, \mathrm{p}<0.01$ ) were significantly higher in patients with CKD than in patients without CKD [15].
Also, in Kelly D.M. et al. study [12] the cardioembolic events rate was higher in patients with CKD (31.8\% versus $21.2 \%$; p $<0.001)$, but this association was not confirmed after adjustment for age, sex, and hypertension. Although patients with CKD had a lower stroke rate due to small vessel pathology $(8.8 \%$ vs. $13.6 \%$; p < 0.001), unknown etiology (26.1\% versus $39.4 \% ; \mathrm{p}<0.001)$ and stroke of another etiology $(1.0 \%$ versus $3.6 \%$; $<0.001)$, these associations were not confirmed after adjustment (adjusted odds ratio $=$ $0.86(0.65-1.13) ; \mathrm{p}=0.27$ and 0.73 (0.36-1.43); $\mathrm{p}=0.37$ for small vessel disease and other defined etiology, respectively) for all but undetermined (adjusted odds ratio $=0.81[0.67-0.98] ; \mathrm{p}=0.03$ ) (odds ratio $=0.81(0.67-0.98) ; \mathrm{p}=0,03)$ [12]. The authors concluded that the renal-specific risk factors are unlikely to play an important role in the etiology of particular subtypes. At the same time, the article emphasizes that the absence of association between CKD and specific TOAST subtypes after adjustment for age and arterial hypertension does not indicate that renal-specific risk factors do not play a role in the cause of all strokes, authors conclude that any such role does not differ in importance between the different etiological subtypes [12].

The reasons for the increased incidence of TOAST2 stroke in CKD group established in our study may be the peculiarities of the cardioembolism pathophysiological mechanisms in reduced renal function conditions, consisting additional factors that predispose the blood clots formation in the left atrium. So, Kizawa S. et al. study [16] showed that CKD can be a significant risk factor for left atrial thrombosis in patients with non-valve atrial fibrillation. A possible association of left atrial thrombogenic milieu (left atrial thrombus, dense spontaneous echo contrast, or left atrial appendage velocity $\leq 25 \mathrm{~cm} / \mathrm{s}$ ) with $\mathrm{CKD}$ in patients with non-valve atrial fibrillation was investigated. Multifactorial logistic regression analysis showed that a decrease in glomerular filtration rate by every 10 $\mathrm{ml} / \mathrm{min} / 1.73 \mathrm{~m}^{2}$ was a significant independent correlate of thrombogenic milieu $(\mathrm{p}=0.005)$, along with nonparoxysmal atrial fibrillation $(\mathrm{p}=0.004)$, a higher score on the CHA2DS2-VASc scale $(\mathrm{p}=0.012)$, every $5 \mathrm{ml} / \mathrm{m}^{2}$ increment in left atrium volume index ( $p<0.001)$, and every $10 \%$ decrement in left ventricular ejection fraction $(\mathrm{p}<0.001)[16]$.

The stroke has deep two-way relationships with chronic kidney disease [5]. Clinicians should pay special attention to the association between CKD and cerebrovascular diseases, to the so-called cerebro-renal interaction [5]. Hypertension, diabetes mellitus and dyslipidemia are the most common risk factors for both cerebrovascular disease and chronic kidney disease. The Bao Y.-S. et al. retrospective research (2013) [13] showed that diabetes mellitus, arterial hypertension, serum uric acid and low-density lipoprotein cholesterol 
(LDL) were independent risk factors for CKD in patients with ischemic stroke [13].

Given that there are common pathogenetic mechanisms, it should be assumed that to one degree or another the cerebrovascular diseases and CKD development progress in parallel, but the development of cerebrovascular diseases is likely significantly accelerate in reduced kidney function conditions. The additional specific CKD pathological conditions, including increased levels of oxidative stress, chronic inflammation, endothelial dysfunction, vascular calcification, anemia, thrombogenic factors caused by the uremic environment, predispose to vascular damage and endotheliopathy [10, 12]. Hypergomocysteinemia, which plays an important role in the malignant atherosclerosis and thrombosis development [9]; lowmolecular $32-\mathrm{M}$ peptide accumulation leading to cerebral amyloid angiopathy development [9] predispose to the cerebrovascular disorders. Accumulation of uremic toxins with reduced kidney function can enhance oxidative stress, chronic inflammation, endothelial dysfunction and vascular calcification [9]. This CKD-specific factors probably predispose to the formation of blood clots in the left atrium and increase the risk of TOAST2 stroke in CKD patients.

The above CKD-specific factors probably contribute to the formation of blood clots in the left atrium and increase the cardioembolic stoke risk. It is also important for understanding of reason leading to TOAST2 increased incidence among CKD patients, that reduced kidney glomerular filtration often limits the medicine use for the stroke prevention and treatment, for example, the oral anticoagulants use for the cardioembolic prevention in atrial fibrillation. The kidney function's condition affects the therapeutic effects of some antithrombotic and thrombolytic drugs, including oral anticoagulants [5].

According to our study data, in IS CKD group the atherothrombotic stroke proportion was lower compared to this one in patients without CKD in the total group (52.5\% versus $66.1 \% ; \mathrm{p}<0.0001 ; \mathrm{OR}=0.565(0.435-0.734)$ ), among male $(54.4 \%$ versus $70.4 \%$; $<<0.0001 ; \mathrm{OR}=0.502$ (0.353-0.713) and among female patients (50.0\% versus $60.1 \% ; \mathrm{p}=0.042 ; \mathrm{OR}=0.665(0.448-0.487))$. The reason for this phenomenon probably is that CKD makes an additional condition to the cardioembolic stroke development in men and women, and it is reflected the increasing TOAST2 proportion and, accordingly, the decreasing TOAST1 proportion in the overall stroke structure. In IS patients without CKD, the TOAST1 proportion was higher in men $(\mathrm{p}=0.004)$, and the TOAST2 proportion was higher in women $(p=0.001)$, at the same time, in the patients with CKD, no significant differences were found in the IS various type frequencies between men and women.
The mean age of IS patients with CKD was statistical significantly higher than mean age of IS patients without CKD: in the general group $68.7 \pm 12.4$ years versus $64.1 \pm 12.5$ years $(p<0.0001)$, among men $-65.7 \pm 12.3$ versus $61.03 \pm 11.6$ years $(\mathrm{p}<0.0001)$, among women $-72.6 \pm 11.6$ versus $68.6 \pm 12.5$ years $(p=0.001)$. This data indirectly supports evidence of a close relationship between CKD and CVD risk factors, which depend on age. These results are consistent with previous studies. Chinda J. et al. [15] also reported that the mean age of CKD stroke patients was higher compared to patients without CKD (75.9 vs. 69.0 years, $\mathrm{p}<0.05$ )

According to our study data, in the disease debut the stroke severity on the NIHSS scale was statistically significantly higher among patients with CKD compared with this one in patients without kidney pathology $(\mathrm{p}=0.0001$ and $\mathrm{p}=0.002$, respectively). Also, the CKD presence affected on the mortality rate. In the total group the mortality rate among CKD patients was higher compared to this one among patients without kidney pathology $(13.7 \%$ vs. $7.4 \%)(\mathrm{p}=0.001$; $\mathrm{OR}=1.979 ; 95 \% \mathrm{CI}: 1.308-2.994)$. According to analyses of stroke type frequencies, among female patients the mortality rate was higher for cardioembolic stroke in CKD patients compared to patients without CKD (29.7\% versus $11.1 \%)(p=0.004$; OR =3.378; $95 \%$ CI: 1.446-7.890), but not for other types of ischemic stroke. At the same time, it was not established any significant differences in mortality rates between the groups with and without CKD among IS male patients. Thus, chronic kidney disease contributed to increased mortality from cardioembolic stroke among women.

The CKD effect on stroke outcome is confirmed by Hayden D. et al. study (2017) [11], in which a decrease in glomerular filtration rate $<45 \mathrm{ml} / \mathrm{min} / 1.73 \mathrm{~m}^{2}$ (hazard ratio $2.53, \mathrm{p}=0.01$ ) was an independent predictor of death in ischemic stroke after 28 days. Poor functional stroke outcome in two years (Rankin modified score of 3-5 points) was more common in patients with renal dysfunction $(52.5 \%$ versus $20.6 \%$, p < 0.001). Thus, impaired kidney function was associated with adverse outcome and lethality [11]

The CKD impact on the hemorrhagic transformation risk in the brain ischemic area may affect the stroke severity. Low glomerular filtration rates were found to be associated with high risk of hemorrhagic transformation in acute ischemic stroke [17]. Lee J.-G. et al. (2013) established a significant association between a glomerular filtration decrease $<30 \mathrm{ml} / \mathrm{min} / 1.73 \mathrm{~m}^{2}$ and hemorrhagic transformation in IS (OR $=2.90 ; 95 \% \mathrm{CI}$ : 1,26-6,68, $\mathrm{p}=0,012$ ). Also, in CKD the hemorrhagic transformation frequency was higher in IS subgroups without thrombolysis (OR $=3.49 ; 95 \% \mathrm{CI}: 1.44-8.46)$ and without atrial fibrillation $(\mathrm{OR}=3.44 ; 95 \% \mathrm{CI}: 1,10-10,76)[17]$. 
Hemodialysis patients have at increased risk of stroke. Among dialysis patients, the prior to stroke risk factors such as arterial hypertension, diabetes mellitus and atrial fibrillation have a significant impact on hospital mortality and outcomes, worsening prognosis [18]. The dialysis impact on stroke risk is supported by the fact that stroke is much more common in dialysis patients than in CKD patients who do not need dialysis [5]. Ischemic and hemorrhagic stroke risk factors among patients with the kidney terminal stage are the dialysisspecific characteristics, such as a hemodynamic dramatic change, dialysate and use of anticoagulants, as well as vascular calcification [5]. The relationship between renal terminal stage and cerebral stroke has a significant impact on morbidity and mortality [18].

Thus, the CKD effecting on the ischemic stroke pathogenetic subtype development has multicomponent etiology, including, traditional CVD factors such as arterial hypertension, diabetes mellitus, dyslipidemia and atherosclerosis, and specific conditions arising from reduced kidney function and leading to vascular endothelial dysfunction, vascular calcification, thrombosis. The combination of these factors predisposes to an increased cardioembolic stroke risk, more severe stroke manifestations, and increased mortality rate.

\section{CONCLUSIONS}

Chronic kidney disease predisposes to the cardioembolic stroke risk, more severe stroke manifestations, and increased mortality rate. Ischemic stroke patients with chronic kidney disease are statistically significantly more likely to develop cardioembolic IS type $(\mathrm{p}<0.0001 ; \mathrm{OR}=1.878 ; 95 \% \mathrm{CI}$ : 1.423-.478), they have more severe stroke manifestations on NIHSS scale $(p=0.0001)$ and on Glasgow scale $(\mathrm{p}=0.002)$, higher mortality rate from cardioembolic stroke in women $(29.7 \%$ versus $11.1 \%)$ $(\mathrm{p}=0.004 ;$ OR $=3.378 ; 95 \%$ CI: 1.446-7.890) compared to IS patients without CKD.

\section{REFERENCES}

[1] D. Kelly, P.M. Rothwell, J. Neurol. Neurosurg. Psychiatry 91(1) (2020) 88-97.

[2] Kidney Disease: Improving Global Outcomes (KDIGO) CKD Work Group, Kidney inter., Suppl. 3 (2013) 1-150.

[3] A.M. Esayan, Med. Council 12 (2017) 18-25.

[4] T. Ninomiya, Contrib. Nephrol. 179 (2013) 58-66.

[5] K. Toyoda, Contrib. Nephrol. 179 (2013) 1-6.

[6] J. Perk, G. De Backer, H. Gohlke et al., Eur. Heart. J. 33 (2012) 1635-1701.
[7] M. Lee, B. Ovbiagele, Expert. Rev. Neurother. 11(2) (2011) 241-249.

[8] K. Toyoda, T. Ninomiya, Lancet Neurol. 13(8) (2014) 823-833.

[9] A.E. Khrulev, A.A. Nikitina, N.S. Khruleva, Cardiovas. therapy and prevent. 18(3) (2019) 8893.

[10] J.M. Chillon, Z.A. Massy, B. Stengel, Nephrol. Dial. Transplant 31(10) (2016) 1606-1614

[11] D. Hayden, C. McCarthy, L. Akijian et al. Int. J. Stroke 12 (2017) 761-769.

[12] D.M. Kelly, Linxin Li, P.M. Rothwell, Oxford Vascular Study, Stroke 51(9) (2020) 2786-2794.

[13] Y.-S. Bao, L.-T. Song, D. Zhong et ak., Eur. J. Clin. Invest. 43(8) (2013) 829-835.

[14] H.P. Adams, B.H. Bendixen, L.J. Kappelle et al., Stroke 24(1) (1993) 35-41.

[15] J. Chinda, N. Nakagawa, M. Kabara et al., Intern. Med. 51 (2012) 1661-1666.

[16] S. Kizawa, T. Ito, K. Akamatsu et al., Am. J. Cardiol., 122 (2018) 2062-2067.

[17] J.-G. Lee, K. B. Lee, I.-M. Jang et al., Cerebrovasc. 35(1) (2013) 53-59.

[18] R. Akku, T.P. Jayaprakash, O.D. Ogbue et al., Cureus. 12(7) (2020) e9484. 